\title{
Indirekte Uebertragung von Varizellen und Varizellen bei einer Erwachsenen.
}

Von Prof. Dr. Otto Lentz, Direktor des Königl. Instituts für Hygiene und Infektionskrankheiten in Saarbrücken.

Krause berichtet in Nr. 19 dieser Wochenschrift über eine Beobachtung von Varizellen bei einem 26jährigen Mädchen.

Er weist dabei auf das verhältnismäßig seltene Vorkommen von Varizellen bei Erwachsenen hin. Da in seinem Falle eine Beruhrung mit Varizellenkranken nicht nachzuweisen war, nimmt Krause an, daß die Uebertragung der Krankheit auf das Mädchen auf indirektem Wege erfolgt sein müsse; er müsse dies annehmen, ,,trotz der entgegengesetzten Ansicht des neuesten Bearbeiters der Varizellen in Nothnagels Pathologie, Ha mburgers, der eine indirekte Infektion nicht zugeben will."

Diese letztere Bemerkung veranlaßt mich, hier kurz eine eigene Beobachtung mitzuteilen, welche 4 Fälle von Varizellen in einer Familie betraf, unter denen einmal ebenfalls eine Erwachsene sich befand und bei denen anderseits die Infektion des ersten Falles einwandfrei auf eine indirekte Uebertragung zurückzuführen war.

B. L., 6 Jahre alt, war als einzige von ihren Geschwistern am 19. März 1909 mit anderen Kindern zusammen bei einer befreundeten Familie zu einer Kindergesellschaft geladen. Als sie von dieser heimkam, spielte sie sogleich mit ihrem $3 / 4$ Jahre alten Schwesterchen G. L. Wenige Tage nachher wurde den Eltern der Kinder von der befreundeten Familie mitgeteilt, daß eines der ebenfalls bei der Kindergesellschaft gewesenen Kinder am 20. März, also am Tage nach der Zusammenkunft mit. B. L., an Windpocken erkrankt sei. Fine weitere Berührung der Kinder mit diesem an Windpocken erkrankten Mädchen hatte nicht stattgefunden; auch sonst waren die Kinder nicht mit windpockenkranken Personen zusammengekommen.

Am 1. April erkrankte nun die 3/4 Jahre alte G. L. an schweren Windpocken; es trat unter Fiebererscheinungen zunächst ein über den ganzen Körper verteiltes, masernartiges Exanthem auf, und auf den Flecken schossen in den nächsten Tagen schubweise spitze Wasserbläschen auf, die allmählich, ohne zu vereitern, eintrockneten und nach etwa drei Wochen ohne Hinterlassung von Narben abheilten.

Am 15. April erkrankte die 3 jährige Schwester R. L. leicht an Windpocken, am 20. April die 29 Jahre alte Mutter der Kinder. Bei dieser waren auf dem Körper und im Gesicht nur vereinzelte Pusteln mit deutlicher Delle vorhanden, gleichwohl war das Befinden bei nur mäßigem Fieber erheblich gestört, und es bestanden starke Kreuzschmerzen; nach der Abheilung der Pusteln blieben im Gesicht zwei deutliche,.Blatternnarben" zurück, von denen eine noch jetzt nach vier Jahren als kleine Narbe erkennbar ist, während die andere vollständig verschwunden ist.

Als letzte endlich erkrankte am 22. April B. L., die offenbar den Infektionsstoff von jener Kindergesellschaft mitgebracht hatte, ohne selbst sofort zu erkranken; sie machte die Krankheit ebenfalls in leichterForm ab.

Da die zuerst erkrankte G. L. noch nicht geimpft war, hätte. man, auch in Anbetracht der starken Ausbreitung der Pockeneruption, an Variola denken können. Hiergegen sprach aber einmal die Form der Pocken, die bei G. L. wie auch ihren Schwestern als ausgesprochene Spitzpocken (ohne Delle) auftraten; ferner sprach dagegen das trotz der starken Ausbreitung des Pockenauschlags über den ganzen Körper verhältnismäßig wenig gestörte Wohlbefinden und völlig freie Sensorium und endlich auch der Ausfall des Tierversuchs. Bei 2 Kaninchen und 1 Meerschweinchen wurde der mittels Kapillaren entnommene Bläscheninhalt in die eingeritzte Hornhaut des rechten Auges eingerieben, während die Hornhaut des linken Auges 
nur in gleicher Weise eingeritzt wurde. Auf allen Augen bildete sich an den Skarifikationsstellen eine ganz leichte Trübung, die gleichmäßig am vierten bis fünften Tage verschwunden war. Bei keinem der Tiere überwog die Veränderung an dem mit dem Bläscheninhalt geimpften Auge wesentlich die des anderen; bei keinem Tiere kam es auch nur zur Andeutung einer Geschwürsbildung.

Der weitere Verlauf der Krankheit bei G. I.. stimmte hiermit überein: die glatte Abheilung der Bläschen ohne Hinterlassung von Narben und der starke Erfolg einer etwa 1/2 Jahr später vorgenommenen Impfung mit Kuhpockenlymphe. Dieser starke Impferfolg sprach auch gegen die sich zunächst aufdrängende Vermutung, daß die schwere Erkrankung bei G. L. ihre Ursache darin hätte haben können, daß das Kind noch nicht geimpft war, während die innerhalb der letzten zwei bis sechs Jahre zum letzten Male geimpften Geschwister und Mutter nur leicht erkrankten, woraus etwa auf eine immunisatorische Beziehung zwischen der Vakzination und den Varizellen hätte geschlossen werden können. Der Unterschied in der Schwere der Erkrankung dürfte wohl vielmehr mit der auch bei anderen Infektionskrankheiten nicht selten gemachten Beobachtung übereinstimmen, da ${ }^{\circ}$ der erste Fall in einer Familie oder auch in einer unter gleichen Bedingungen lebenden Menschengruppe (z. B. in einer Kaserne, einem Alumnat u. ä.) der schwerste ist die sich anschließenden Fälle dagegen leichter verlaufen.

Bemerkenswert ist ferner, da B bei der Mutter der Kinder die Pockeneruption trotz ihrer geringen Ausbreitung bei fast fieberlosem Verlauf doch mit einer ziemlich erheblichen subjektiven Störung des Allgemeinbefindens und mit starken Kreuzschmerzen einherging, einem Symptom, das sonst als charakteristisch für Variola gilt, das aber nach meinen Erfahrungen differentialdiagnostisch nur verwertet werden kann, wenn es fehlt, da sein Fehlen gegen echte Pocken spricht.

Als das wichtigste Moment meiner Beobachtung erscheint mir aber der Umstand, daß hier einwandfrei festgestellt werden konnte, daß die Infektion des ersten Falles der kleinen Kontaktreihe auf indirektem Wege durch eine gesunde Person erfolgte, die zunächst auch gesund blieb und erst im weiteren Verlauf der Epidemie, jedenfalls erst infolge direkten Kontaktes mit der zuerst erkrankten Schwester, erkrankte. Man darf annehmen, daß, wie hier durch eine gesunde Person eine Uebertragung der Varizellen vermittelt wurde. auch durch infizierte Gegenstände der Krankheitskeim einmal indirekt übertragen werden kann.

Zusammenfassung. Es konnte die indirekte Uebertragung von Varizellen durch Vermittlung einer gesunden Person einwandfrei festgestellt werden. Bei dem zuerst erkrankten Kinde infizierte sich dessen 29jährige Mutter, die das bei Varizellen sehr seltene, sonst für Variola als ganz charakteristisch angesehene Symptom ".Kreuzschmerzen" aufwies. 